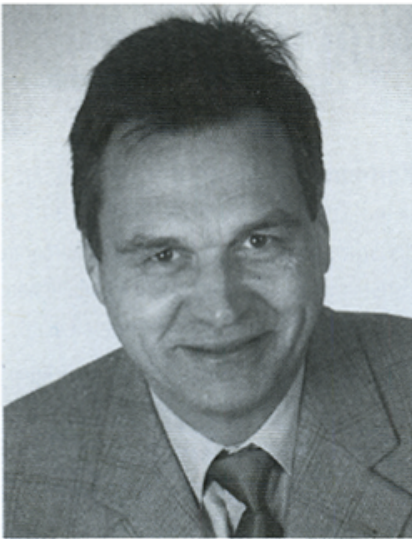

Uwe Fichtner

\section{Modelle von Welten}

zu entwerfen, Rahmenbedingungen für ihre Abbildung zu formulieren und sie für den Nutzer zu gestalten, das ist die gemeinsame Klammer der drei Aufsätze in diesem Heft, die in ihrer Unterschiedlichkeit nicht nur einen breiten fachlichen, sondern auch interessanten zeitgeschichtlichen und wissenschaftstheoretischen Bogen spannen. Sie demonstrieren, dass Fragen der Orientierung und des Sichzurechtfindens für den Menschen von grundlegender Natur sind und deshalb für alle Situationen gelten, in denen er sich Räume aneignet, deren Gestalt und Abbilder entwirft und sie für sich in Gebrauch nimmt, egal, ob es sich dabei um reale oder virtuelle Welten handelt. An dieser Nahtstelle lenkend in die technische Entwicklung und den gesellschaftlichen Wandel einzugreifen, visuell überzeugende und zugleich verlässliche Antworten anzubieten und zu liefern, gehört ohne Zweifel zu den Grundaufgaben der Kartographie, die von ihr zu allen Zeiten gefordert wurde - weshalb man auch die von Sven Fuhrmann und Alan M. MacEachren behandelte Thematik keineswegs als Spielerei abtun darf, sondern ihre große theoretische Reichweite und praktische Auswirkung auf unser Fach im Blick haben sollte. Beim Aufbau von Modellen dieser Art rücken verstärkt messtechnische Probleme in den Vordergrund: Wie reliabel sind denn die Informationen, die der Nutzer auf diese Weise gewinnt und inwieweit besitzen die vermittelten Inhalte Validität?

Kein GIS kann heutzutage ohne Weltkoordinaten auskommen und ihre Projektion mit Hilfe von Netzentwürfen ist beim Umgang mit Karten unverzichtbar. Erst explizit formulierte Abbildungsverfahren, wie hier von Kurt Bretterbauer an einem speziellen Beispiel vorgeführt und erläutert, bilden bekanntlich eine wissenschaftliche, d.h. nachvollziehbare, jederzeit überprüfbare und widerspruchsfreie Basis für die Verwendungsmöglichkeiten und den Einsatz von Karten. Diese Anforderung muss ebenso für eine Modellierung virtueller Welten in wissenschaftlichem Umfeld gelten.
Der Beitrag von Günter Hell vermittelt insbesondere auch GIS-Anwendern grundlegendes methodisches Wissen, da es zu den im Alltag ständig wiederkehrenden Notwendigkeiten gehört, dass Informationen aus unterschiedlichen Karten zusammengeführt und ein einmal gewählter Netzentwurf geändert werden muss.

Den status-quo in der Erfassung und Wiedergabe von Karten markiert und dokumentiert Walter Stupp mit seinem Bericht. Er gibt gewissermaßen einen Kurzabriss über die rasante Entwicklung der Reprotechnik von der analogen zur digitalen Technik mit ihren enweiterten Einsatzmöglichkeiten.

Darüber hinaus wird die verehrte Leserschaft gebeten, ihre Aufmerksamkeit der honorigen Internationalen CoronelliGesellschaft zuzuwenden. Besonders zahlreich fallen in diesem Frühjahr die laudationes von Lebensleistung und Werk herausragender Persönlichkeiten aus, die sich um die Kartographie verdient gemacht haben. Darunter findet sich so manches lesenswerte Detail mit zahlreichen Rückblicken und vielen, für den Werdegang von Kartographen typischen Schnittpunkten.

Die im letzten Editorial gemachte Andeutung, in der DGFK sei zurzeit viel Dynamik, wurde fast umgehend bestätigt: Im Laufe seiner Sitzung am 19. Mai 2001 in Berlin kam der Vorstand der DGfK einvernehmlich zu der Ansicht, für eine Bewältigung der anstehenden und komplexen Zukunftsaufgaben sei es am besten, den Weg für eine neve Mannschaft frei zu machen, und trat geschlossen zurück. Es ist beabsichtigt, anlässlich der Mitgliederversammlung auf dem 50. DKT in Berchtesgaden einen neven Vorstand zu wählen. An alle Mitglieder der DGfK richtet sich deshalb die dringende Bitte, die Erklärung auf S. 159 in diesem Heft und den zugehörigen Aufruf des Wahlleiters zu beachten.

Ihr

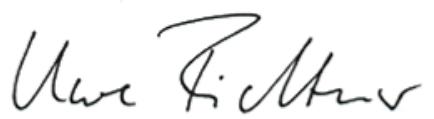

